\title{
Applying social network analysis to input-output based innovation matrices: an illustrative application to six OECD technological systems for the middle '90s
}

\author{
Sandro Montresor* Giuseppe Vittucci Marzetti ${ }^{\dagger}$
}

January 15, 2009

\begin{abstract}
The paper discusses, illustrates and possibly contributes to overcome two methodological problems which emerge in applying Social Network Analysis (SNA) to the study of IO-based innovation flows matrices. The first one has to do with the scale-effects these matrices suffer from. The second one refers to the need of dichotomising the matrices. Through an illustrative application to 6 OECD countries in the mid-'90s, the paper shows that, as for the former problem, different relativisation procedures can be and has been used, which either tend to alter the actual meaning of standard SNA indicators, or do not properly take into account the actual composition of countries' final demand. As for the latter problem, the paper shows that the choice of discrete cut-offs is extremely sensitive, as comparative results actually change along the continuous of the matrices values. In order to overcome the scale problem, a new relativisation procedure is put forward which measures innovation flows embodied in a unit value basket of final demand and thus properly retains all the information provided by the original matrix of intersectoral innovation (embodied) flows. In addressing the problem of dichotomisation, the paper suggests, as a second best, to work with density distributions which can make the choice of discrete cut-off values less arbitrary.
\end{abstract}

\footnotetext{
*Department of Economics, Univ. of Bologna, Italy. E-mail: sandro.montresor@unibo.it
}

${ }^{\dagger}$ Department of Economics, Univ. of Trento, Italy. E-mail: giuseppe.vittucci3@unitn.it 
Keywords Technological systems; Embodied innovation flows; InputOutput analysis; Network analysis

JEL Classification: O33; O38; O39 


\section{Introduction}

Although it gained popularity in the early '90s, the system analysis of the innovation process has recently undergone an important transformation. The need of complementing qualitative analyses of those institutions and organizations which make up systems of innovation - national, sub-national, super-national and regional - with rigorous quantitative measurements and evaluations of their relationships, structure and performance, has spurred to look for new and more suitable methodological tools.

Among these, as DeBresson (1996a) shew so brightly with his long research career, the analysis of economic interdependence represents an extremely powerful tool of investigation. Indeed, combining Input-Output (IO) analysis with that of innovation activity, and building up intersectoral innovation flows matrices, provides the researcher with a precious map trough which to investigate the constitutive relationships of a system of innovation. What is more, this map can be fruitfully explored by using network analysis. Sectors and intersectoral flows can be in fact dealt with as the constitutive elements of innovation networks whose properties can be investigated through Social Network Analysis (SNA). As has been shown by a number of studies (see, for instance, DeBresson, 1996a; Leoncini and Montresor, 2003b; Chang and Shih, 2005; Montresor and Vittucci Marzetti, 2008), several SNA indicators have an immediate meaning in the system analysis of innovation. For example, the density of the network identified by intersectoral innovation flow matrices - defined as the ratio between the number of innovation flows of a certain significance and the total number of possible flows - can be taken as a proxy of the actual connectivity degree of a certain innovation system: the higher the density, the more actually cohesive - i.e. "systemic" - the system is.

With respect to this research program, the present paper intends to provide a methodological value added, by discussing two issues in applying SNA to IO-based innovation flows matrices. The first issue is that of the scale-effects these matrices suffer from. In absence of a proper relativisation, matrices of absolute innovation flows are affected by the scale of the sectoral innovative efforts of the correspondent innovation system. Indeed, by construction, the most innovation-intensive systems turn out to be also the most cohesive in terms of absolute innovation flows, so that a direct structural comparison is misleading. In order to avoid this problem, different relativisation procedures have been applied. However, their implications for the 
relative network analysis have not been sufficiently addressed: something this paper intends to do, also by putting forward a new, alternative relativisation procedure.

The second problem in applying SNA to innovation flows matrices has to do with the need of dichotomising the matrices, once made them relative, in order to apply a good part of the most significant SNA indicators. A need which involves the choice of a cut-off value - with respect to which the actual innovation flows are compared in order to turn the relative cells into " $1 \mathrm{~s}$ ", if greater, or " $0 \mathrm{~s}$ ", if lower - which the results of the analysis crucially depend on. In this last respect, the paper intends to provide an illustrative application of the great sensitivity of the results of SNA from the selected threshold values: a problem which can be partially accommodated by working with density distributions and possibly overcome by looking at new network analysis techniques.

The structure of the paper is the following. Section 2 sketches the theoretical background of the paper. Section 3 discusses the scale and the dichotomisation issue and the problem of obtaining proper intersectoral innovation flows to work with. Section 3.1 critically reviews previously proposed relativisation procedures and compares them with some new methodological proposals, while Section 3.2 does the same with respect to dichotimisation by suggesting to work with density distributions. Section 4 sets these procedures at work in an application carried out by comparing the technological systems of 6 structurally different OECD countries (Japan, Korea, Netherlands, Poland, Spain, USA) in the middle '90s, and tries to get signs of systematic vs. occasional differences. Section 5 concludes.

\section{Theoretical background: investigating technological systems through SNA of innovation matrices}

The methodological issues this paper addresses are typically faced in comparing the technological systems of two or more countries (or sectors) by looking at their intersectoral innovation flows. ${ }^{1}$

\footnotetext{
${ }^{1}$ More in general, the same issue is relevant whenever two or more countries are compared on the basis of a certain intersectoral matrix which distributes the sectoral values of a certain variable along their subsystem structure. The comparative analysis of
} 
This research program, which has recently attracted a number of studies (see, for instance, DeBresson, 1996a; Leoncini and Montresor, 2003b; Chang and Shih, 2005), draws on the following 4 theoretical points.

(i) The innovation process does not occur among (atomistic) economic agents acting in isolation. Rather, it is innervated and shaped by a set of relationships through which innovative institutions and organisations (e.g. firms, research labs, universities, patent offices, and the like) interact, learn and originate different kinds of what have been called Systems of Innovation (SI) (e.g. Lundvall, 1992; Edquist, 1997; Edquist and McKelvey, 2000).

(ii) Not only are innovative relationships institutional and affected by the topographical, geographical space in which they operate (think of a firm localised close to a research lab), but they are also technical and economic in their nature, that is also affected by a techno-economic kind of space, which is the matching of: "an abstract economic space of supply and demand of different goods (as represented by input-output matrices) and [a] technical space (as represented by the techno-functional classification of patents)" (DeBresson, 1996b, p.151). Indeed, a firm in the motor-vehicles sector is closer to one in the mechanical, rather than in the textile sector, irrespectively from their actual geographical distance. In order to retain both the spaces, a broad notion of Technological System (TS) is required, which also retains production kind of relationships (Leoncini, 1998; Leoncini and Montresor, 2003a), as from the following point.

(iii) The constitutive relationships of a TS can be proxied and mapped by building up a suitable matrix of intersectoral innovation flows, namely, a $(n \times n)$ matrix of R\&D flows embodied in vertically integrated sectors such as the following:

$$
\mathbf{R}=\hat{\mathbf{r}} \hat{\mathbf{q}}^{-1}(\mathbf{I}-\mathbf{A})^{-1} \hat{\mathbf{y}}=\hat{\mathbf{r}} \mathbf{B}
$$

where $\hat{\mathbf{r}}, \hat{\mathbf{q}}$, and $\hat{\mathbf{y}}$ are the diagonalised vectors of, respectively, sectoral $\mathrm{R} \& \mathrm{D}$ expenditures, gross production and final demand, and $(\mathbf{I}-\mathbf{A})^{-1}$ is the Leontief inverse matrix based on domestic input coefficients (A) (e.g. Marengo and Sterlacchini, 1990).

The generic element of this matrix, $R_{i j}$, measures the amount of $\mathrm{R} \& \mathrm{D}$ invested by sector $i$, an thus approximately of its innovations, which is

deindustrialisation and tertiarisation processes is thus another potential field of application for it (Montresor and Vittucci Marzetti, 2007). 
embodied in the production flows required to it by sector $j$ in order to satisfy, directly and indirectly, one unit of its final demand. Given the way it is defined, such a matrix actually reflects the functioning of both the innovative sub-system (proxied by R\&D expenditure) and the production sub-system (proxied by IO tables) of a TS. Furthermore, the resort to the operator $\mathbf{B}$ in reclassifying $\mathrm{R} \& \mathrm{D}$ flows from pure sectors into vertically integrated sectors (or subsystems) allows the same flows to retain also the market side of a TS through the role of final demand. ${ }^{2}$

(iv) The structure of a TS and the different role of its constitutive sectors can be analysed, especially in a cross-country comparative framework, by applying to intersectoral innovation matrices suitable Social Network Analysis (SNA) techniques and indicators (Leoncini, Maggioni, and Montresor, 1996; Leoncini and Montresor, 2000a,b). Indeed, the economic sectors and the intersectoral techno-economic flows of these matrices can be envisaged as, respectively, the vertices (or nodes) and the arcs (or directed edges) of the constitutive network of the correspondent TS. In particular, three SNA indicators are particularly useful in comparing different TSs. ${ }^{3}$

The first one refers to the TS as a whole, and is the density $(\delta)$ of the correspondent network of $n$ sectors, defined as:

$$
\delta(t)=\frac{\sum_{i} \sum_{j(i \neq j)} d_{i j}(t)}{n(n-1)} \text { with } 0 \leq \delta(t) \leq 1
$$

where $d_{i j} \in\{0,1\}$ is the element of the so called "contingency matrix" $\mathbf{D}(t)$, amounting to the dichotomic transformation - according to a certain cut-off $t$ - of the matrix $\mathbf{R}$ or, as we will argue in the next section, of a suitable relative transformation of it (an issue on which we will return later). The technoeconomic interpretation of $\delta(t)$ with respect to a TS is quite immediate: the larger (the smaller) is $\delta(t)$, the more (the less) dense is the network, the more (the less) connected (i.e. systemic) is the correspondent TS.

Unlike the previous one, the second SNA indicator refers to each vertex

\footnotetext{
${ }^{2}$ The B operator was first proposed by Siniscalco (1982). Each row of it adds up to 1 and shows "the shares of output of each sector which contribute to the different subsystems". Thus, B can be used to reclassify any physical or value magnitude from sectors into subsystems. As noted by Rampa (1982), it is relative price invariant and depends not only on strict technological factors, but also on the structure of final demand.

${ }^{3}$ As Montresor and Vittucci Marzetti (2008) show, the same kind of analysis can be fruitfully employed also to detected and investigate different forms of innovation clusters within the TSs.
} 
$j$ of a network, and is given by its degree, or more precisely, indegree and outdegree centrality. This indicator is nothing but the number of vertices through which a certain node $j$ is reached by (indegree) and reaches (outdegree) the other $n-1$ vertices of the network. In a directed graph, such as that correspondent to the intersectoral innovation matrices we are dealing with, the indegree and outdegree centrality of a vertex $j$ are distinct and formally defined as follows:

$$
\begin{gathered}
C_{j}^{\text {in }}(t)=\sum_{i(i \neq j)} d_{i j}(t) \text { with } 0 \leq C_{j}^{i n}(t) \leq n-1 \\
C_{j}^{\text {out }}(t)=\sum_{j(j \neq i)} d_{i j}(t) \text { with } 0 \leq C_{j}^{\text {out }}(t) \leq n-1
\end{gathered}
$$

where, as before, $t$ refers to a certain cut-off value. When dealing with TSs, given that inward and outward arcs represent, respectively, intersectoral innovative acquisitions and diffusions, the two measures of centrality can help determining the degree of dependency or pervasiveness of the sectors in a TS.

The last SNA indicator we consider refers to the TS as a whole again, and is given by the degree centralisation, or more precisely, indegree and outdegree centralisation of the correspondent network, defined as:

$$
H^{i n}(t)=\frac{\sum_{j}\left(C_{j^{*}}^{i n}(t)-C_{j}^{i n}(t)\right)}{(n-1)^{2}} \text { with } 0 \leq H^{i n}(t) \leq 1
$$

$$
H^{\text {out }}(t)=\frac{\sum_{j}\left(C_{j^{*}}^{\text {out }}(t)-C_{j}^{\text {out }}(t)\right)}{(n-1)^{2}} \text { with } 0 \leq H^{\text {out }}(t) \leq 1
$$

where $C_{j^{*}}^{i n}$ and $C_{j^{*}}^{\text {out }}$ are, respectively, the indegree and outdegree centrality of the most central vertex $j^{*}$.

As low values of the centralisation identifies a network with similar (centrality) positions, the correspondent TS can be deemed one in which its sectoral partitions are "evenly distributed". Conversely, large centralisation values refer to TSs with highly "hierarchic" sectoral partitions (Leoncini and Montresor, 2000b). 


\section{3 "Scale" and "cut-off" in comparing different TSs: two delicate issues}

Although the interpretative power of a network (intersectoral) analysis of different TSs is quite high, this can be obtained only by dealing satisfactorily with two crucial methodological problems.

\subsection{Getting rid of scale effects: alternative relativisation procedures}

The first problem refers to the choice of the proper "relative" intersectoral innovation flows matrix to which the network analysis should be then applied. Indeed, although the need of transforming the absolute values of the $\mathbf{R}$ matrices - through which the correspondent TSs are compared - into relatives ones might appear intuitive, the actual way such relative matrices are obtained is far from innocuous: conversely, as we intend to show with this paper, the results of a network intersectoral analysis of the TSs are quite sensitive to it.

First of all, given the way $\mathbf{R}$ is defined in Equation 1, it is straightforward that applying the SNA indicators directly to the $\mathbf{R}$ matrices of a certain number, $z$, TSs to be compared - even when expressing the correspondent vectors of R\&D expenditure in PPP terms - will end out by yielding misleading results. Should country 1 be much larger (in economic terms) than country 2 , and thus have a larger scale of R\&D activities across all the considered sectors, the density analysis would show, a fortiori, higher values for the former than for the latter country with respect to all the cut-offs. However, concluding that TS1 is more connected than TS2 would be, in this case, not guaranteed: although less consistent, and thus systematically excluded from the dichotomisation, the embodied sectoral R\&D flows of the latter might be more consistent in relation to the overall scale of the economic activity of the system they refer to, but by using absolute R\&D flows we are unable to capture it.

In order to get rid of scale effects, and make density and the other SNA indicators more robust in informing about the relational structure of the compared TSs, we need to normalise the absolute intersectoral flows of $\mathbf{R}$ in some way. But with respect to what? Indeed, scale differences across different TSs could be traced at different levels. Not only could the scale of R\&D activities of different TSs be systematically different, but 
two TSs could have similar R\&D scales and, still, systematically different demand volumes - spurring differently the intersectoral innovation flows in a sub-system framework - or different production volumes - conveying differently the intersectoral innovation flows embodied in them. What is more, considering that TSs have also a sectoral dimension, along with a national one (Malerba, 2004), there could be systematic scale differences across different subsystems. In the light of these considerations, rather than one relativisation procedure only, different ways of getting normalised intersectoral flows should be considered. As we will show in the following, these different procedures yield different results, so that their choice should be based on an accurate evaluation of their pros and cons and, above all, of their rationale.

Although the outcome is always represented by some kind of relative matrix, different relativisation procedures can be accomplished, depending on the scale of operation one wants to get rid of and on the national or sector/subsystem focus of the analysis.

\subsubsection{Unit value matrix}

A first possible relativisation procedure is that proposed by Chang and Shih (2005), who suggest to compare the intersectoral structure of different TSs by resorting to a unit value matrix $\left(\mathbf{R}^{\text {unit }}\right)$ defined as follows: ${ }^{4}$

$$
\mathbf{R}^{\text {unit }}=\hat{\mathbf{r}} \hat{\mathbf{q}}^{-1}(\mathbf{I}-\mathbf{A})^{-1}
$$

Let us observe that using this matrix amounts to dividing each column of the original matrix $\mathbf{R}$ by the final demand level of the correspondent subsystem, that is:

$$
\mathbf{R}^{\text {unit }}=\mathbf{R} \hat{\mathbf{y}}^{-1}
$$

Accordingly, we can conclude that the different subsystems of each TS are in this way scaled down to the same unit final output, irrespectively from

\footnotetext{
${ }^{4}$ Although Chang and Shih (2005) convert all the values in US dollars, this does not prove strictly necessary. Indeed, denoting with $E$ the nominal exchange rate of the home currency to the US dollar, we have that the matrix obtained by using $E\left(\mathbf{R}_{E}^{\text {unit }}\right)$ is equivalent to that without $\left(\mathbf{R}^{\text {unit }}\right)$ :
}

$$
\mathbf{R}^{\text {unit }}=\hat{\mathbf{r}} \hat{\mathbf{q}}^{-1}(\mathbf{I}-\mathbf{A})^{-1}=E^{-1} \hat{\mathbf{r}} \hat{\mathbf{q}}^{-1} E\left(\mathbf{I}-E^{-1} \mathbf{A} E\right)^{-1}=\mathbf{R}_{E}^{\text {unit }}
$$


the ratios between the original operational scales of the different subsystems, which could be different in different TSs.

In the light of this latter fact, the use of $\mathbf{R}^{\text {unit }}$ entails a focus on the individual subsystems of each TS. Therefore, this procedure might be justified when one is interested in the TS conceived as a distinguishable "constellation" of individual subsystems. On the other hand, let us observe that $\mathbf{R}^{\text {unit }}$, while it gets rid of the differences in countries' overall GDP - which is desirable - it also neutralises countries' differences in its structure - which is not as desirable. ${ }^{5}$

\subsubsection{Unit basket of final demand}

In order to retain the different structure of the final demand in the compared TSs, while still getting rid of overall scale differences, we suggest to resort to a different relativisation procedure, and work out the following matrix:

$$
\mathbf{R}^{\text {basket }}=\frac{1}{\mathbf{i}^{\prime} \mathbf{y}} \mathbf{R}
$$

where $\mathbf{i}^{\prime}$ is a unit row vector.

The matrix $\mathbf{R}^{\text {basket }}$ calculates the innovation flows embodied in the intermediate production ones "activated" by a unit basket of final demand. Thus, while it still scales down the different subsystems to a comparable cross-country level, it leaves unaltered the operational scale ratios between the different subsystems of each TS. Just to give an example, in the case of a fictitious economy made up of three sectors, whose final demand vector is $\mathbf{y}=(1500,2500,1000)$, the correspondent unit basket of final demand will be $(0.3,0.5,0.2)$, unlike $(1,1,1)$ as for $\mathbf{R}^{\text {unit }}$.

\subsubsection{Normalised $\mathbf{R}$}

Although the previous relativisation removes cross-country differences connected with the "size" of their economy, their relative ranking in terms of aggregate $R \& D / G D P$ ratios still affects the network analysis which is based on it.

If one is interested in focusing on the purely relational characteristics of the different TSs, that is, on how embodied innovation flows are distributed

\footnotetext{
${ }^{5}$ It is also worth noting that such method tends to underestimate the weight of the R\&D efforts of the less developed countries, unless a PPP correction is introduced.
} 
among the different subsystems within each TS, irrespectively from the value of such flows as a proportion of the overall size of the system, he needs to move to a different relativisation. In this case, a possible relativisation procedure we suggest could be the simple normalisation to one of the $\mathbf{R}$ matrices, that is:

$$
\mathbf{R}^{\text {norm }}=\frac{1}{\mathbf{i}^{\prime} \mathbf{R} \mathbf{i}} \mathbf{R}=\frac{1}{\mathbf{i}^{\prime} \mathbf{r}} \mathbf{R}
$$

Indeed, the ratio between each element of $\mathbf{R}^{\text {basket }}\left(r_{i j}^{\text {basket }}\right)$ and the correspondent element of $\mathbf{R}^{\text {norm }}\left(r_{i j}^{n o r m}\right)$ is constant and equal to the country's aggregate $R \& D / G D P$ ratio:

$$
\frac{r_{i j}^{\text {basket }}}{r_{i j}^{\text {norm }}}=\frac{r_{i j}}{\mathbf{i}^{\prime} \mathbf{y}} / \frac{r_{i j}}{\mathbf{i}^{\prime} \mathbf{r}}=\frac{\mathbf{i}^{\prime} \mathbf{r}}{\mathbf{i}^{\prime} \mathbf{y}} \quad(i, j=1,2, \ldots, n)
$$

\subsubsection{Matrix C}

A last possible method to relativise the matrix $\mathbf{R}$ is that of normalising the subsystem innovative acquisitions, that is of dividing each cell of the original matrix by the sum of the correspondent column, thus building up a matrix C defined as follows:

$$
\mathbf{C}=\mathbf{R}\left(\widehat{\mathbf{i}^{\prime} \mathbf{R}}\right)^{-1}
$$

where the hat symbol is used to denote diagonalisation. ${ }^{6}$

This matrix, put forward by Leoncini, Maggioni, and Montresor (1996), has been used by Leoncini and Montresor (2000b, 2003b, 2005) and, more recently, by Montresor and Vittucci Marzetti (2008) for is "mixed" properties. On the one hand, like $\mathbf{R}^{\text {norm }}, \mathbf{C}$ emphasises "pure" relational aspects. On the other hand, unlike $\mathbf{R}^{\text {norm }}$, but like $\mathbf{R}^{\text {unit }}$, the focus is kept on the individual subsystems of each TS.

\subsubsection{A synthesis}

In brief, the four relativisation procedures described above combine two different levels of analysis:

\footnotetext{
${ }^{6}$ It is worth noting that, given the way $\mathbf{R}$ is defined, dividing its cells by the sum by row simply returns the operator $\mathbf{B}$; in formal terms:
}

$$
(\widehat{\mathbf{R i}})^{-1} \mathbf{R}=(\widehat{\mathbf{r}} \widehat{\mathbf{B}} \mathbf{i})^{-1} \hat{\mathbf{r}} \mathbf{B}=(\widehat{\hat{\mathbf{r}} \mathbf{i}})^{-1} \hat{\mathbf{r}} \mathbf{B}=\hat{\mathbf{r}}^{-1} \hat{\mathbf{r}} \mathbf{B}=\mathbf{B}
$$


Table 1: Rationale of the relativisation procedures

\begin{tabular}{llll}
\hline \multirow{2}{*}{ Relativisation dimension } & \multicolumn{2}{l}{ Focus of the analysis } \\
\cline { 2 - 4 } & & System & Subsystem \\
\cline { 2 - 4 } & Economic & $\mathbf{R}^{\text {basket }}$ & $\mathbf{R}^{\text {unit }}$ \\
& Technological & $\mathbf{R}^{\text {norm }}$ & $\mathbf{C}$ \\
\hline
\end{tabular}

(i) the first level concerns the dimension along which one might want to get rid of scale effects, that is, economic activity (proxied by the volume of final demand) or technological activity (proxied by R\&D expenditure);

(ii) the second dimension refers instead to the focus of the analysis one wants to carry out through relative matrices, that is, system-focus or subsystem-focus (see Table 1)

On principle, $\mathbf{R}^{\text {basket }}$ and $\mathbf{R}^{\text {norm }}$ appear more consistent with the inner logic of the TS analysis. However, looking the TS as an "artificial constellation" of subsystems might be preferable when one is interested in recovering, also and above all, their different centrality in different TSs. As we will argue in the following, the choice of one of the four procedures should be inspired by the research questions one intends to address. Thus, there is no one absolute best, or worst, procedure among them.

\subsection{Getting dichotomic matrices: density distributions and cut-off values}

The second problem is possibly more well-known than the former, and due to the fact that the SNA indicators described in Section 2 require us to work with dichotomic matrices, that is matrices made up of $1 s$ and $0 s$ depending on the values of the retained matrices of intersectoral innovation flows being, respectively, larger and smaller than a certain cut-off value $t$. Indeed, as one can easily figure out, and as we will show in our application, the results are sensitive to the choice of the relevant cut-off value and thus in principle not robust, at least in absolute terms.

Of course, in order to solve this problem in toto, one could avoid using binary data at all, and applying SNA indicators directly to one of the four relative versions of the matrix $\mathbf{R}$ presented in Section 3.1. In this last respect, several progresses have been made in the SNA literature in recent years, such as: the use of weighted adjacency matrices to analyse complex networks 
(Onnela, Saramaki, Kertész, and Kaski, 2005; Barthélemy, Barrat, PastorSatorras, and Vespignani, 2005), where the node degree is substituted by the node strength and suitable generalizations of some of the indicators used in SNA - e.g. clustering coefficient (e.g. Saramäki, Kivelä, Onnela, Kaski, and Kertész, 2007; Fagiolo, 2006) and betweenness centrality (Newman, 2005) - have been provided $;^{7}$ or the recent temptative extensions of generalized blockmodeling (Doreian, Batagelj, and Ferligoj, 2005) to valued networks (e.g. Ziberna, 2007).

These and other methods of treating the value matrices, such as $\mathbf{R}$, in SNA are for sure extremely useful in carrying out, on the basis of them, quantitative, "univariate" analyses of the impact that intersectoral innovation diffusion has on the economic performance of the investigated TSs. Typically, in building up intersectoral R\&D stocks from what are called intersectoral $R \mathscr{E} D$ spillovers, and in estimating econometrically their impact on a certain productivity and/or productivity growth measure (e.g. Mohnen, 1997; van Meijl, 1997; Hanel, 2000; Dietzenbacher and Los, 2002).

On the other hand, the same techniques are not superior to the standard ones when the aim of the research is that of identifying and mapping the relational structure of the TSs under analysis. An empirical application which, although possibly more qualitative, is not less relevant than the previous ones. The endogenous identification of innovation clusters (Montresor and Vittucci Marzetti, 2008), if not even of "reduced" systems of innovation (OECD, 1999, 2001), within nation-wide TSs, typically carried out by investigating the graphs generated by the $\mathbf{R}$ matrices, is the most policy-relevant example of such a kind of empirical application: indeed, it turns out to be crucial in detecting in which part of the techno-economic space a certain $R \& D$ and/or innovation policy should be applied and have the most pervasive effects. In this and similar other applications, the resort to dichotomic matrices allows to obtain important information even by making economies of more sophisticated SNA techniques. The best one can do in these cases is to avoid choosing the cutoffs in a purely arbitrary way, and rather base their choice on comparisons and at least partial sensitivity analyses.

One way of proceeding according to this rationale has been suggested by Leoncini and Montresor (2000b), who started working with more cut-off

\footnotetext{
${ }^{7}$ Recent empirical applications of this complex weighted-network analysis are Fagiolo, Schiavo, and Reyes (2008); De Montis, Barthélemy, Chessa, and Vespignani (2007).
} 
values through a two-step procedure: (i) for each of the $z$ TSs to be compared, building up a density distribution $\mathbf{d}\left(\mathbf{t}_{z^{*}}\right)$ using as cut-off values the ordered distribution, $\mathbf{t}_{z^{*}}$, of the $n \times(n-1)$ cells of a proper reference matrix, that is of a reference TS, $z^{*}$; (ii) by comparing the $z$ density distributions, extracting "heuristically" some cut-off values out of the distribution $\mathbf{t}_{z^{*}}$, with respect to which carrying out the rest of the network analysis and checking for their robustness. In a recent paper, Montresor and Vittucci Marzetti (2008) refined this procedure by suggesting to run step (i) with respect to a "super-vector" of cut-offs $\left(\mathbf{t}_{Z}\right)$, whose $Z$ elements are obtained by ordering the cells of all the $z$ matrices to be compared $(Z=(n \times(n-1)) \times z)$.

As we will see, while the application of this technique clearly shows the dependence of the SNA results from the selected cut-offs, on the other hand, it at least signals, when they exist, subsets of cut-off values with respect to which they could be retained relatively robust.

\section{An application to six OECD technological systems for the middle '90s}

In order to grasp the implications of the two methodological issues debated above, in the following we present the results of an empirical application carrying out by comparing, on a relational basis, six OECD TSs for the middle '90s. Although only apparently descriptive, this application is, in the spirit of evolutionary economics, functional to what Nelson and Winter (1982) called "appreciative theorising", that is to the identification of observed phenomena for which the researcher is then asked to find explanations and causal links. In this case, the phenomena we want to shed light on is the actual system nature of six TSs and the different role that different economic sectors have on them (Leoncini and Montresor, 2003b): phenomena that the analysis of the productivity growth impact of $R \& D$ spillovers and of other research strands instead inspired by "formal theorising" should take into account.

From an operational point of view, by using IO data taken from the OECD Input-Output Database (2005) and crossing them with data on sectoral R\&D expenditure (OECD ANBERD Database, 2005), we have worked out the $\mathbf{R}$ matrix (Equation (1)) with respect to 6 OECD countries, chosen out of 15 available, by crossing the criterion of data availability with that of having sufficient heterogeneity among the correspondent TSs, as docu- 
mented by other qualitative studies (e.g Nelson, 1993), that is: Japan, Korea, Netherlands, Poland, Spain and the USA. ${ }^{8}$

With respect to these TSs (i.e. $\mathbf{R}$ matrices), the four relativisation procedures have been used to obtained four different benchmarks, with respect to which we have then applied the dichotomisation method discussed in Section 3.1. In other words, for each relativisation procedure, each TS has been transformed into a series of 1440 dichotomised matrices $(\mathbf{D}(t))$, one for each element of the "super-vector" of cut-offs $\left(\mathbf{t}_{Z}\right)^{9}$, and with respect to these matrices the TS distributions obtained by applying the network indicators discussed in Section 2 have been worked out.

In so doing, it is therefore possible to analyse how the different network indicators are distributed for each relativisation procedure.

\subsection{Density distributions}

To start with, let us consider how the relative distribution of the six TSs changes in moving from one relativisation method to the other when their densities (Equation (2)) are considered (Figure 1). By applying the first method (Section 3.1.1), Japan appears the densest TS all along the range of cut-off, followed by the USA, Korea and, at a distant, Netherlands, Spain and Poland (Figure 1(a)). There is practically no inversion in this ranking and one can therefore conclude that, no matter which is the chosen cut-off, Japan is at least as dense as all the other countries in the sample, thus, the most connected TS among the chosen ones. On the contrary, Poland is the least connected TS. At first sight, this was the picture one could expect by thinking of what we know about these TSs in qualitative and quantitative terms (Leoncini and Montresor, 2003b; OECD, 2003). Working with this first relativisation procedure, therefore, one could confidentially investigate the impact of the cohesiveness of these TSs on their performance without having to bother too much with the selected cut-off.

However, when the structure of the final demand is considered, that is, by applying the second relativisation method (Section 3.1.2), the ranking

\footnotetext{
${ }^{8}$ Although matrix inversions have been carried out for each country at the maximum level of disaggregation in order to reduce the distortions introduced by sectoral aggregation, the blanks in the series of the latter dataset have forced us to limit our empirical application to 16 sectors only (see Appendix A for details).

${ }^{9}$ As we have said, the $\mathrm{Z}$ elements of $\mathbf{t}_{Z}$ are obtained by ordering the cells of all the 6 relativised matrices, so that $Z=(15 \times 16) \times 6=1440$.
} 


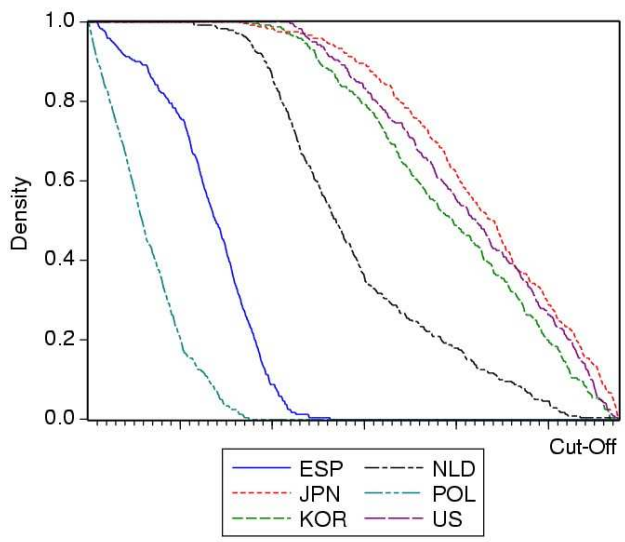

(a) Unit value matrices

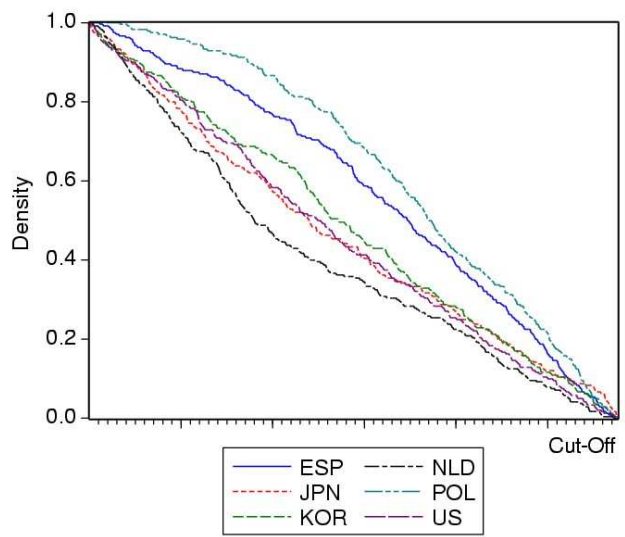

(c) Normalised $\mathbf{R}$

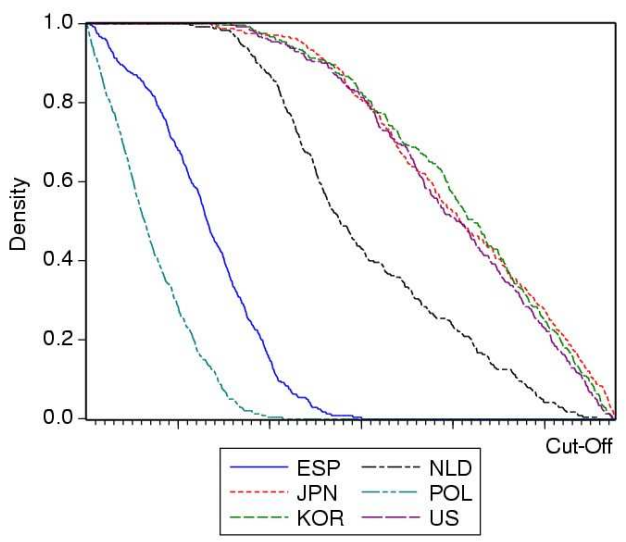

(b) Unit basket of final demand matrices

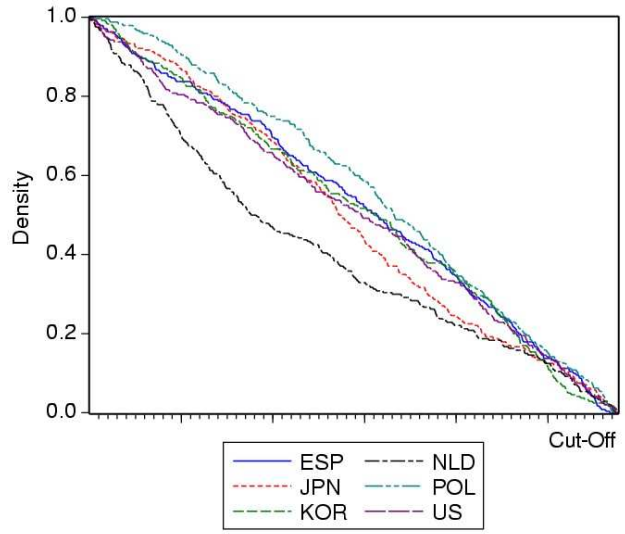

(d) Matrices $\mathbf{C}$

Figure 1: Density distributions for the different relativisation methods

of the densest TSs becomes more blurred, and the sensitivity to the cut-off value increases. General results can still be appreciated, such as the fact that Korea jumps to the role of the densest TS over a quite wide range of cut-off values (Figure 1(b)). Thus, taking into account the proportion in which the different subsystems are actually operated within each country, that is, retaining also the market sub-system in the TS characterisation, does make difference. The substantial improvement of Korea's position with the second method might also be partly due to the underestimation of the contribution of R\&D efforts for developing countries introduced by the former method if no PPP correction is used. And this bias should be taken into account when 
economic systems with different levels of development are compared. ${ }^{10}$

By looking at the previous density distributions, one is also legitimated to suspect that the low ranking of Poland and Spain, although after having discounted the different scale of their economy, is still due to the low aggregate $\mathrm{R} \& \mathrm{D} / \mathrm{GDP}$ ratios compared to the others. Discounting this further aggregate factor, and confronting the different TSs on the ground of their "pure" relational aspects, is however possible. The density distributions of Figure 1(c), obtained by working with normalised $\mathbf{R}$ matrices (Section 3.1.3), actually show that this can radically change the overall picture. Indeed, quite surprisingly, now Poland and Spain rank, respectively, first and second all along the range, while the least connected TS becomes Netherlands. This means that the former two TSs, once discounted for their relatively low level of aggregate R\&D expenditure, turn out to be in fact highly connected. On the contrary, in the Netherlands, also neglecting the quite low R\&D/GDP ratio characterising such country with respect to Japan, Korea and the USA, the TS turns out to be only weakly connected, so to say "structurally". This is an extremely important result in the system analysis of innovation. As has been also shown in other intersectoral analyses (e.g. Montresor and Vittucci Marzetti, 2008), working with standard innovation related variables, such as sectoral or total $R \& D$ intensity, even by retaining and cumulating the role of intersectoral spillovers, might not provide a complete picture of a TS: although intensively low, extensively its innovativeness could be high, a result which could affect the application of industrial and innovation policies.

As for as $\mathbf{R}^{\text {norm }}$ is concerned, it is worth emphasising that, although the sum of its elements is equal to one by definition, that of the elements out of the main diagonal can range from 0 to 1 . Thus, the TS density distributions worked out from this matrix actually results from: on the one side, the weight of the intersectoral embodied innovation flows on the intrasectoral ones; on the other side, the distribution of such intersectoral flows among the different sectors. As shown by Figure 2, for all the six analysed countries but Japan, the ranking in terms of weight of intersectoral innovation flows over intrasectoral ones corresponds to that derived from the analysis of countries' density distributions. ${ }^{11}$ Japan is instead an exception

\footnotetext{
${ }^{10}$ For the delicate issue of R\&D data on PPP see also Dougherty, Inklaar, McGuckin, and van Ark (2007).

${ }^{11}$ In passing, let us note that intersectoral embodied flows are quite low compared to the intrasectoral ones, amounting on average for the six countries to $18 \%$ of the total flows,
} 


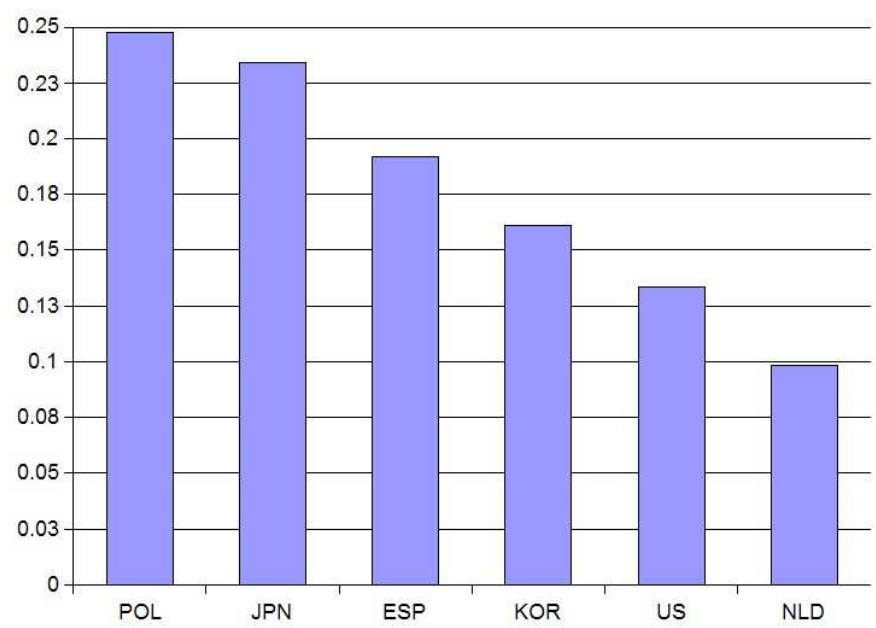

Figure 2: Weight of intersectoral flows on total embodied innovation flows

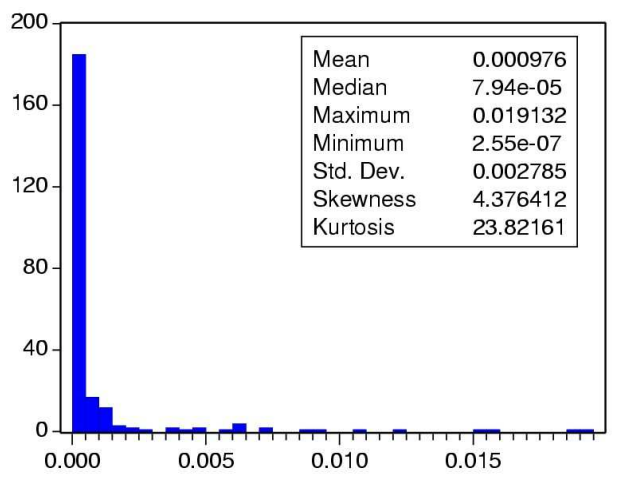

(a) Japan

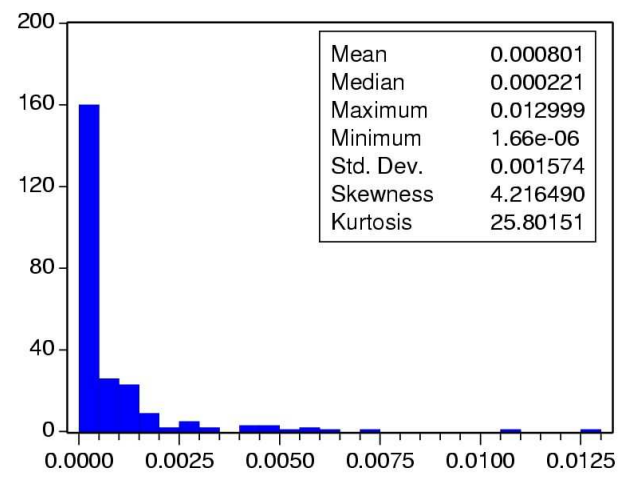

(b) Spain

Figure 3: Distributions of intersectoral flows in normalised $\mathbf{R}$

suggesting that, although intersectoral flows are quite high in absolute terms ( $23.4 \%$ of the total), they are apparently more polarised in relative terms, thus leading to the low ranking of the correspondent TS in Figure 1(c). The simple comparison of the distribution of the extradiagonal values for Japan and Spain confirms, in terms of basic indicators, this result (Figure 3).

The density analysis is concluded by Figure 1(d), which shows the density distributions obtained by using the matrix $\mathbf{C}$ of normalised subsystem innovative acquisitions (Section 3.1.4). This time, instead of being relativised with respect to the total innovation flows of the correspondent TS, the inward with a coefficient of variation of $32.6 \%$. 


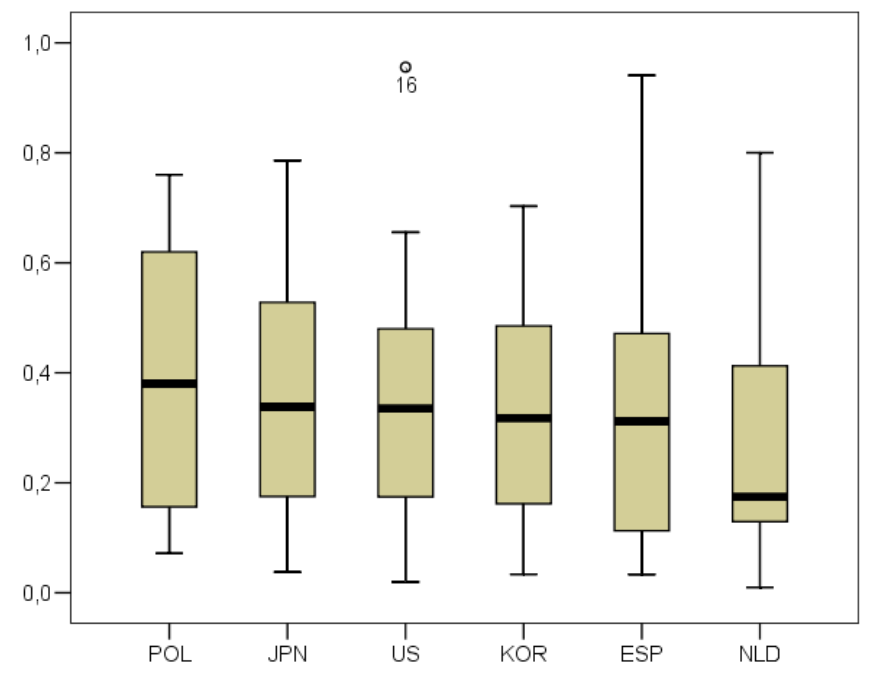

Figure 4: Distributions of the weights of intersectoral innovative acquisitions on total subsystem acquisitions

flows of each subsystem are normalised with respect to its own total acquisitions. Thus, each subsystem is treated in relative isolation. Accordingly, what can in fact affect the density distributions of each TS as a whole is not as much the ratio between its intrasectoral and intersectoral total embodied innovation flows, as in the former method. But rather the weight of the innovative acquisitions each subsystem gets from the other sectors on the total inward flows of the subsystem itself.

Figure 4 reports the box-plots of the distributions of these weights for the different TSs and shows that the TS density distributions of Figure 1(d) can be partly explained by the distribution of such weights. For example, the most (least) structurally dense TS, that is Poland (Netherlands), actually shows the highest (lowest) ordered statistics when compared with the others. ${ }^{12}$

By using $\mathbf{C}$, the general picture provided by Figure 1(c) gets somewhat confirmed, but the TS density distributions become more similar. When using this relativisation procedure, therefore, one has to be aware of the fact

\footnotetext{
${ }^{12}$ However, it is worth noting that, assuming normality - not rejected at the $10 \%$ significance level ( $5 \%$ for Netherlands) by the Kolmogorov-Smirnov test - and variance homogeneity across the different groups - not rejected by the Levene statistic $(\mathrm{F}(5,90)=.244$, $\mathrm{p}$-value $=.942)-$, the ANOVA test does not reject the null hypothesis of mean equality of these weights across the different TSs $(\mathrm{F}(5,90)=.354$, p-value $=.88)$, and this result does not change with non parametric statistics (Kruskal Wallis test p-value $=.794$ ).
} 
that the dichotomisation problem becomes more relevant. Furthermore, it has to be emphasised that, given the main subsystem focus of this method, when one wants to derive features related to the overall TS, as something different from the sum of its subsystems, relativising $\mathbf{R}$ matrices with such procedure turns out to be not fully appropriate. ${ }^{13}$

\subsection{Centralisation distributions}

Not only do the relativisation procedures discussed above affect density, but also the other network indicators. This is particularly true when their impact on the indegree and outdegree centralisation (see Section 2) distributions is considered. In particular, as shown by Figures 5 and 6 , the relativisation procedures affect the absolute and local maximums of these indicators as well as the relative ranking of the TSs according to them. ${ }^{14}$

At the outset, it should be noted that, while the density distributions are quite different - such as when one deals with TSs showing remarkable differences in their aggregate $\mathrm{R} \& \mathrm{D} / \mathrm{GDP}$ ratio - by applying one of the first two relativisation methods (Figures 1(a) and 1(b)) the relative degree centralisation distributions are not "centered", and this can make cross-TS comparisons in terms of centralisation very sensible to the cut-off which is actually chosen (Figures 5(a), 5(b), 6(a) and 6(b)).

In this case, therefore, rather than comparing the TS centralisation at the same cut-off, as for the density analysis, a sounder choice would be to compare the absolute and local maximums of the indicator for the different TSs. In other words, in the present case, centralisation would serve to answer different research questions: irrespectively from the chosen threshold flow-size (i.e. cut-off), which might be different in the different TS to be compared, which is the maximum value of degree centralisation attained by each TS? Which is the relative differences in such maximums? For which flow-size difference with respect to such maximum is a certain TS equally hierarchical from another one? Indeed, these are questions which deserve attention in the system analysis of innovation, and which can be significantly addressed

\footnotetext{
${ }^{13}$ In this respect, Chang and Shih (2005) are right when they point out that this relativisation procedure is "unable to produce a comparable base for displaying the differences between [...] countries" (2005, p.157), although it has to be stressed that, as seen before, such remark can be equally applied to their method too.

${ }^{14}$ Given the way it is defined, the degree centralisation, inward or outward, tends to 0 when the density of the correspondent network tends to 1 or 0 .
} 


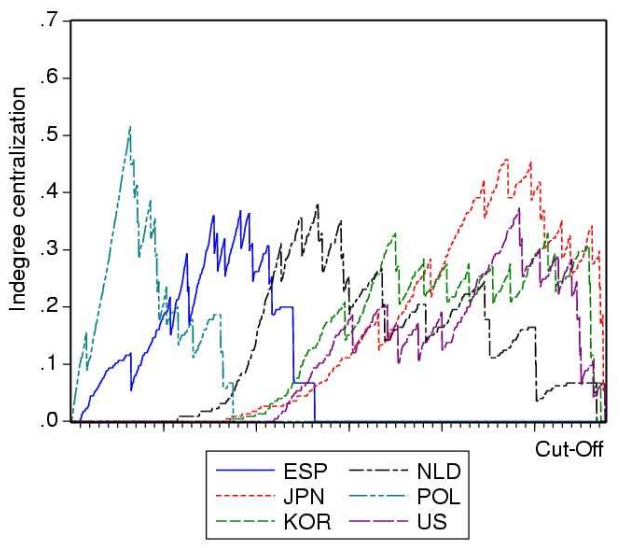

(a) Unit value matrices

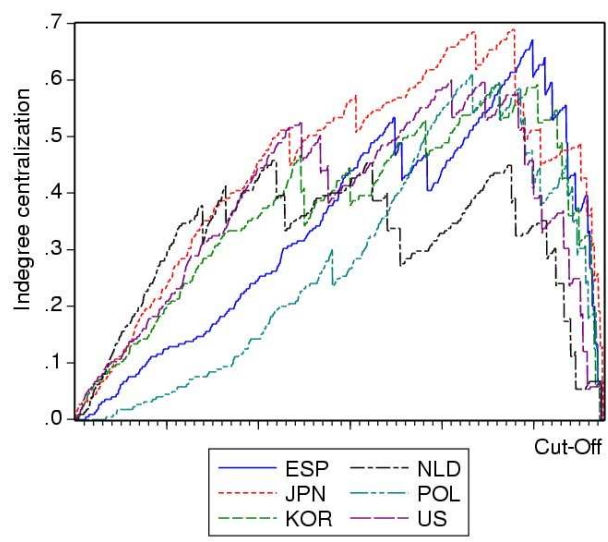

(c) Normalised $\mathbf{R}$

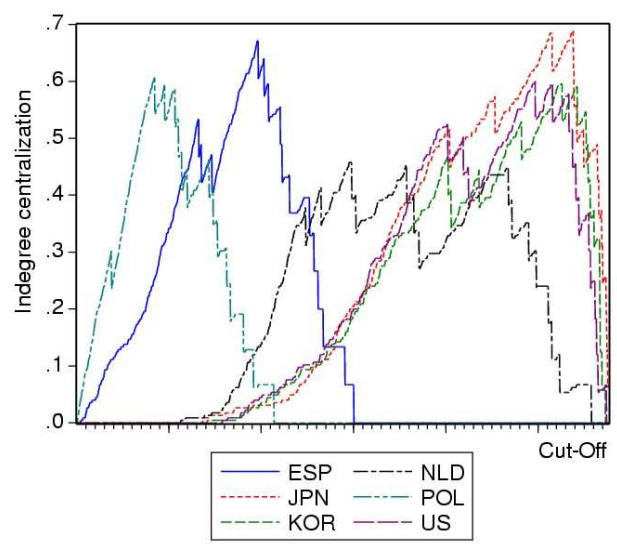

(b) Unit basket of final demand matrices

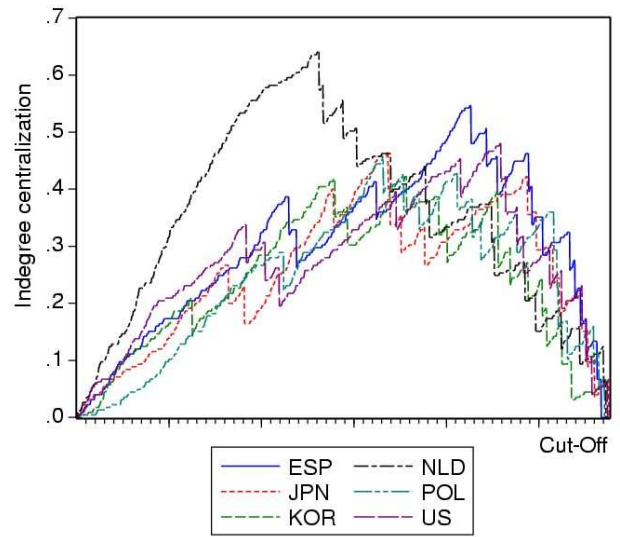

(d) Matrices $\mathbf{C}$

Figure 5: Indegree centralisation distributions for the different relativisation methods

even in the presence of a strong sensitiveness to the cut-off values.

Although this holds true for both $\mathbf{R}^{\text {unit }}$ and $\mathbf{R}^{\text {basket }}$, which of the two is actually chosen could be decided on the ground of their inner logic (Section 3.1.5): when the indicator refers to the TSs as a whole, as degree centralisation does, and the latter are not simply seen as a constellation of individual subsystems, the second method should be preferred (Figures 5(c) and 6(c)).

As far as the third relativisation procedure is concerned, that is $\mathbf{R}^{\text {norm }}$, an interesting relationship with $\mathbf{R}^{\text {basket }}$ should be noticed. Given that, for each TS, the ratio between every element of $\mathbf{R}^{\text {basket }}$ and the correspondent element of $\mathbf{R}^{\text {norm }}$ is constant and equal to country's aggregate R\&D/GDP 


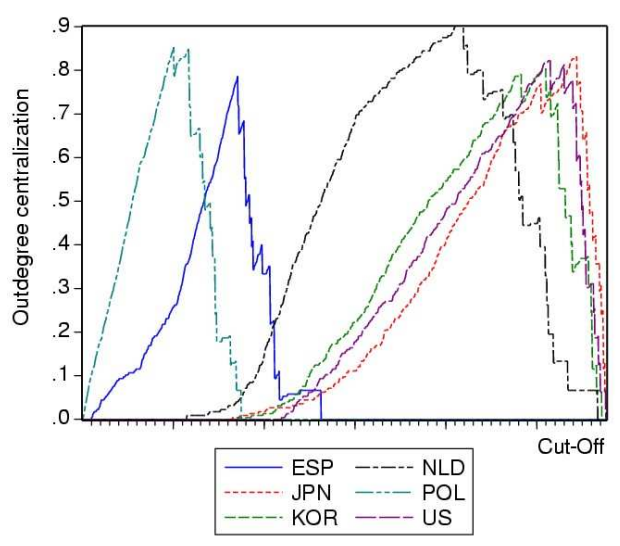

(a) Unit value matrices

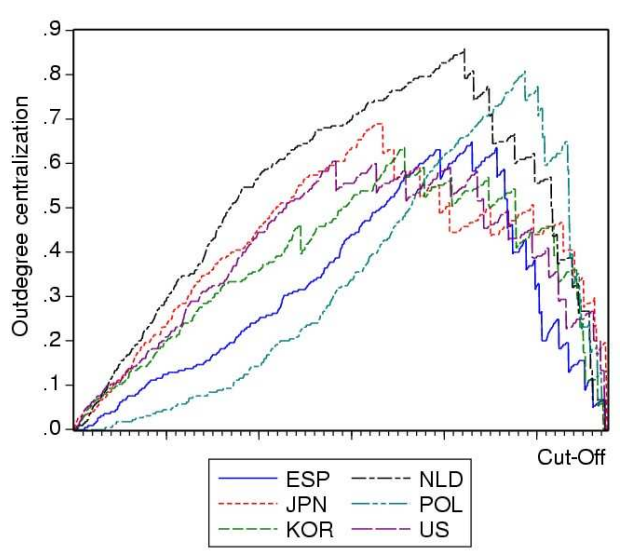

(c) Normalised $\mathbf{R}$

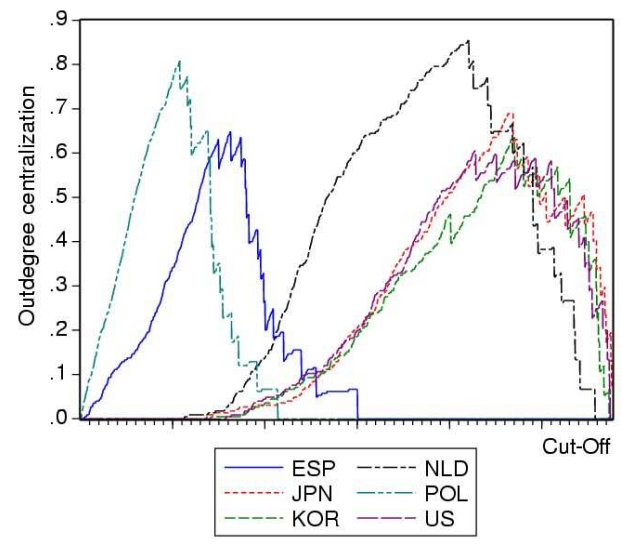

(b) Unit basket of final demand matrices

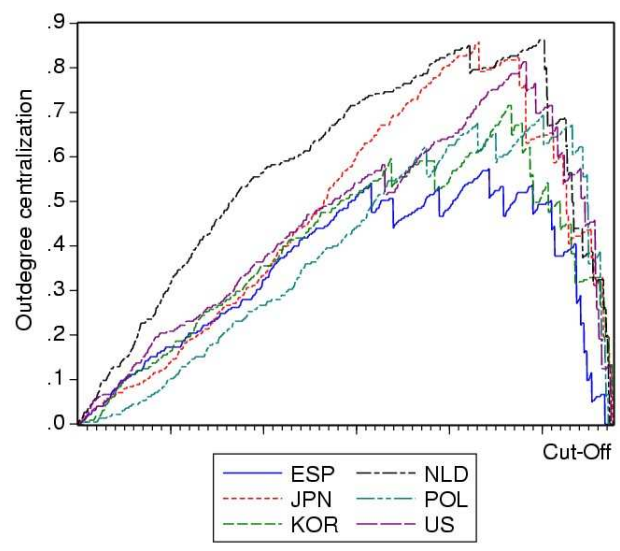

(d) Matrices $\mathbf{C}$

Figure 6: Outdegree centralisation distributions for the different relativisation methods

ratio (Equation (11)), applying the latter procedure does not alter the centralisation ranking of absolute and local maximums worked out with the former, but simply "centralises" the TSs's distributions. As this makes the TSs more easily comparable (Figures 5(c) and 6(c)) in terms of centralisation, and does not affect the centralisation values, when the latter are the focus (irrespectively of the correspondent cut-off) $\mathbf{R}^{\text {norm }}$ should thus be preferred.

Following the same line of reasoning, and with the same research question in mind, one can argue that this method should be preferred also to the last one (Figures 5(d) and 6(d)). Indeed, although by using the matrix $\mathbf{C}$ one can still obtain "centralised" distributions, the main focus of the method on 
the individual subsystems should prevent its application when the analysis is mainly concerned with the whole TSs.

A last comment should be spent on the TS centralisation distributions when indegree rather than outdegree centralisation indicators are used. By comparing Figure 5 and 6 it appears immediately that, in spite of the same relativisation arguments, the TS rankings in the two cases are different. Just to make an example, when outdegree centralisation is considered, the Netherlands emerge for the highest values and this, apart from the low density of this TS, seemingly reveals a high dependency on few key sectors for innovation diffusion. Conversely, by looking at indegree centralisation, it is Japan which stands out, revealing the presence in its TS of one or more sectors which are dependent on the rest of the TS much more than the average.

More in general, the different meaning of indegree and outdegree centralisation should be clearly disentangled, whatever relativisation procedure is adopted.

\subsection{Centrality distributions}

Given the close relationship with the correspondent centralisation indicators, the impact of the alternative relativisation procedures on the last network indicator presented in Section 2, that is, degree centrality, does not require much further exploration. In order to provide an illustration, we have chosen to focus on textiles (Sector 4), out of the 16 available subsystems we have for each TS (Appendix A), and decided to accomplish the analysis only with respect to the indegree centrality (Figure 7).

At the outset, we should notice that, although this sector is structurally dependent in technological terms, as the majority of the applied innovation studies reveal from the times of the famous Pavitt taxonomy (Pavitt, 1984), its indegree centrality is quite different across the six TSs for a number of cut-offs. What is more important, the relativisation procedure crucially alters the centrality gap of the sector across the TSs, so that the relative choice has to be carefully thought also in this last respect.

Following the criteria set in Section 3.1, the relativisation methods more suitable for the analysis of degree centrality should be the first $\left(\mathbf{R}^{\text {unit }}\right)$ and the last one $(\mathbf{C})$. Indeed, we are now comparing a specific subsystem in different TSs, thus, the relativisation procedure should be able to discount 


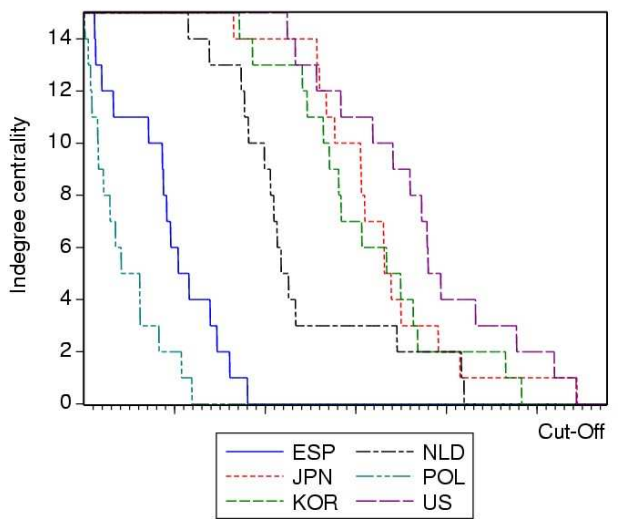

(a) Unit value matrices

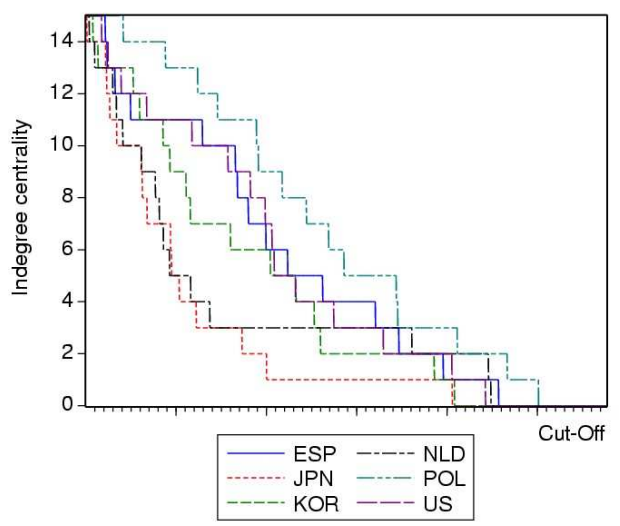

(c) Normalised $\mathbf{R}$

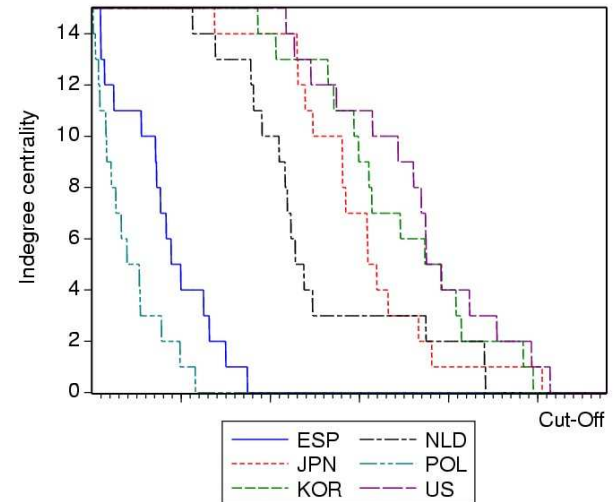

(b) Unit basket of final demand matrices

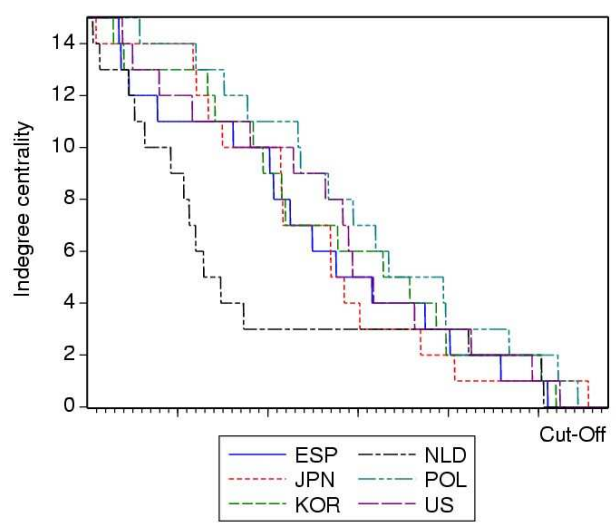

(d) Matrices $\mathbf{C}$

Figure 7: Indegree centrality distributions of textiles for the different relativisation methods

for all the factors more related to the TS it belongs to, rather than to the subsystem itself. ${ }^{15}$

In so doing, it is possible just to rescale the subsystem to the same operational scale in all the TSs (Figure 7(a)) or normalising to one its total embodied innovation inflows (Figure $7(\mathrm{~d})$ ). Once more, let us notice that working with the $\mathbf{C}$ substantially increases the problem of the cut-off sensitiveness, as the 6 centrality distributions largely overlap in continuous switching and re-switching. On the other hand, working with $\mathbf{R}^{\text {unit }}$ more general result emerge. For example, quite interestingly, the American textile

\footnotetext{
${ }^{15}$ It has to be stressed that the situation would be different if one instead had to compare two or more subsystems within the same TS in order to infer their relative position in it.
} 
sector is by far the most dependent in innovation terms by the other sectors of the TS, while the least dependent among the 6 compared is the Polish one, and this quite irrespectively from the selected cut-off. On the contrary, the indegree centrality of the textile sector in Korea and in Japan actually appears structurally similar all along the cut-offs, possibly suggesting a certain socio-cultural characterisation of the relative sectoral system of innovation.

\section{Conclusions}

The paper has discussed two methodological issues which emerge in applying SNA to IO-based innovation matrices which inform about the relational nature of nation-wide TSs.

The first one is that of getting rid of scale effects, in order to be able to compare different TSs from a structural point of view. In this last respect the paper has argued, and showed in its empirical application, that different relativisation procedures yield different results when the main network indicators are applied. In particular, differences have to do with the aspect the researcher is interested to focus on in comparing different TSs. For example, by scaling the innovation matrices with respect to the $\mathbf{R}^{\text {unit }}$ matrix, and thus just discounting the different innovation intensity of the different national subsystems, Japan actually appeared in the middle '90s the densest TS of the six compared, as suggested by other more qualitative studies (e.g. Nelson, 1993; Leoncini and Montresor, 2000a). On the other hand, however, by working with $\mathbf{R}^{\text {norm }}$, and thus focusing on purely relational innovative aspects, Poland and Spain appear as the most systemic, though less innovation intensive TSs.

The second issue the paper focuses on is that of dichotomising the original value matrices in order to apply those tools of network analysis which cannot be directly applied to value graphs and, in general, to carry out qualitative analyses of the intersectoral maps and clusters which can be identified in different TSs. When, such as in these cases, working with binary data is unavoidable, the paper suggests to start the analysis from, and indeed base it on, the distributions of SNA indicators for the compared TSs. Indeed, as the empirical application has shown, this methodology enables the researcher to understand when the sensitiveness of the results to the selected cut-off is quite low, and then the correspondent outcome of a certain robustness. For 
example, when the dichotomisation is undertaken on the basis of $\mathbf{R}^{\text {unit }}$, the different centrality that the textile sector has in two such different countries as US and Poland is a result which does not depend on it. On the contrary, in some other cases, working with distributions might suggest the researcher not to work at the level of individual cut-offs, and rather concentrate on the distribution extremes and extreme differentials across the TSs, as the application has proved to be case with respect to the centralisation analysis.

Both these issues should be carefully retained, first of all, in innovation analyses which, although of qualitative nature, have a lot of policy implications, such as the endogenous identification of innovation clusters (Montresor and Vittucci Marzetti, 2008), if not even of "reduced" systems of innovation (OECD, 1999, 2001). But the same holds true also for those, possibly more quantitative analyses, which use the former ones as input, such as that of the productivity and productivity growth impact of intersectoral R\&D spillovers.

In concluding the paper, we would like to stress once more that, although mainly intended to address the methodological issues encountered in the comparative analysis of TSs through intersectoral innovation flows matrices, the achieved results turn out to have a broader set of applications. Indeed, they can be fruitfully applied each time one deals with value matrices representing input and output flows which have to be somehow relativised in order to remove "scale" effects, whatever they are, and then dichotomised by fixing a certain threshold in order to be able to use the great majority of network analysis tools. The analysis of intersectoral "knowledge flows" is just an example of this kind of extensions.

\section{References}

Barthélemy, M., A. Barrat, R. Pastor-Satorras, and A. VespigNANI (2005): "Characterization and modeling of complex weighted networks," Physica A, 346, 34-43.

Chang, P.-L., and H.-Y. Shin (2005): "Comparing patterns of intersectoral innovation diffusion in Taiwan and China: A network analysis," Technovation, 25(2), 155-169.

De Montis, A., M. Barthélemy, A. Chessa, and A. Vespignani (2007): "The structure of Inter-Urban traffic: A weighted network analysis," Environment and Planning: B, 34, 905-924. 
DeBresson, C. (1996a): "The Inter-industrial analysis of innovative activities," in Economic Interdependence and Innovative Activity. An InputOutput Analysis, ed. by C. DeBresson, pp. 66-78. Edward Elgar, Cheltenham.

(1996b): "Why innovative activities cluster," in Economic Interdependence and Innovative Activity. An Input-Output Analysis, ed. by C. DeBresson, pp. 149-165. Edward Elgar, Cheltenham.

Dietzenbacher, E., And B. Los (2002): "Externalities of R\&D Expenditures," Economic Systems Research, 14(4), 407-425.

Doreian, P., V. Batagelu, and A. Ferligoj (2005): Generalized Blockmodeling. Cambridge University Press.

Dougherty, S. M., R. Inklaar, R. H. McGuckin, and B. van Ark (2007): "International Comparisons of R\&D Expenditure: Does an R\&D PPP Make a Difference?," Working Paper 12829, National Bureau of Economic Research, Cambridge, MA.

Edquist, C. (ed.) (1997): Systems of Innovation. Technologies, Institutions and Organisations. Pinter Publishers, Londra.

Edquist, C., And M. McKelvey (eds.) (2000): Systems of Innovation: Growth, Competitiveness and Employment. Edward Elgar Publishing, Cheltenham, UK.

FAgiolo, G. (2006): "Clustering in Complex Directed Networks," mimeo.

Fagiolo, G., S. Schiavo, and J. Reyes (2008): "On the topological properties of the world trade web: A weighted network analysis," Physica A.

Hanel, P. (2000): "R\&D, Interindustry and International Technology Spillovers and the Total Factor Productivity Growth of Manufacturing Industries in Canada, 1974-1989," Economic Systems Research, 12(3), $345-361$.

LEONCINI, R. (1998): "The nature of long-run technological change: innovation, evolution and technological systems," Research Policy, 27, 75-93. 
Leoncini, R., A. Maggioni, and S. Montresor (1996): "Intersectoral innovation flows and national technological systems: network analysis for comparing Italy and Germany," Research Policy, 25, 415-430.

LeOncini, R., And S. Montresor (2000a): "Classifying technological systems: an empirical application to eight OECD countries," in Technology and Knowledge: from the Firm to Innovation Systems, ed. by P. Saviotti, and N. B., pp. 152-173. Edward Elgar, Cheltenham.

(2000b): "Network Analysis of Eight Technological Systems," International Review of Applied Economics, 14(2), 213-234.

(2003a): "The technological system," in Technological Systems and Intersectoral Innovation Flows, ed. by R. Leoncini, and S. Montresor, pp. 33-49. Edward Elgar, Cheltenham.

(2003b): Technological Systems and Intersectoral Innovation Flows. Edward Elgar, Cheltenham.

(2005): "Accounting for Core and Extra-Core Relationships in Technological Systems: a Methodological Proposal," Research Policy, 34, 83-100.

Lundvall, B.-A. (ed.) (1992): National Systems of Innovation. Towards a Theory of Innovation and Interactive Learning. Pinter, London.

Malerba, F. (ed.) (2004): Sectoral Systems of Innovation: Concepts, Issues and Analyses of Six Major Sectors in Europe. Cambridge University Press, Cambridge.

Marengo, L., and A. Sterlacchini (1990): "Intersectoral technology flows. Methodological aspects and empirical applications," Metroeconomica, 41, 19-39.

Mohnen, P. (1997): "Introduction: Input-Output Analysis of Interindustry R\&D Spillovers," Economic Systems Research, 9(1), 3-8.

Montresor, S., and G. Vittucci Marzetti (2007): "The deindustrialisation/tertiarisation hypothesis reconsidered: a subsystem application to the OECD7," mimeo. 
(2008): "Innovation Clusters in Technological Systems: a Network Analysis of 15 OECD Countries for the Middle '90s," Industry $\& 3$ Innovation, 15(3), 319-345.

Nelson, R. (ed.) (1993): National Innovation Systems. A Comparative Analysis. Oxford University Press, Oxford.

Nelson, R., And S. Winter (1982): “An Evolutionary Theory of Economic Change," .

Newman, M. E. J. (2005): "A measure of betweenness centrality based on random walks," Social Networks, 27, 39-54.

OECD (1999): Boosting innovations, the cluster approach. OECD, Paris.

(2001): Innovative clusters, drivers of national innovation systems. OECD, Paris.

OECD (2003): OECD Science, Technology and Industry Scoreboard 2003 Towards a knowledge-based economy. OECD, Paris.

Onnela, J., J. Saramaki, J. Kertész, and K. Kaski (2005): "Intensity and coherence of motifs in weighted complex networks," Physical Review E, 71, 065103 .

PAvitt, K. (1984): "Sectoral Patterns of Technical Change: Towards a Taxonomy and a Theory," Research Policy, (13), 343-373.

Rampa, G. (1982): "Commento a Momigliano e Siniscalco," Moneta e Credito, 139, 475-479.

Saramäki, J., M. Kivelä, J. Onnela, K. Kaski, and J. Kertész (2007): "Generalizations of the clustering coefficient to weighted complex networks," Physical Review E, 75(2), 27105.

SiniscalCO, D. (1982): "Il Sistema Produttivo: Analisi per Industrie e Subsistemi," Ricerche Economiche, XXXVI(4), 475-488.

VAn MeiJl, H. (1997): "Measuring Intersectoral Spillovers: French Evidence," Economic Systems Research, 9(1), 25-46.

ZibernA, A. (2007): "Generalized blockmodeling of valued networks," Social Networks, 29(1), $105-126$. 


\section{A Sector classification}

\begin{tabular}{clc}
\hline & Sector & ISIC Rev.3 Code \\
\hline 1 & Food products, beverages and tobacco & $15-16$ \\
2 & Textiles, textile products, leather and footwear & $17-19$ \\
3 & Wood, paper, printing, publishing & $20-22$ \\
4 & Coke, refined petroleum products and nuclear fuel & 23 \\
5 & Chemicals (including pharmaceuticals) & 24 \\
6 & Rubber and plastics products & 25 \\
7 & Other non-metallic mineral products & 26 \\
8 & Basic metals & 27 \\
9 & Fabricated metal products (except machinery and equipment) & 28 \\
10 & Machinery and equipment, nec & 29 \\
11 & Electrical and optical instruments & $30-33$ \\
12 & Motor vehicles, trailers and semitrailers & 34 \\
13 & Other transport equipment & 35 \\
14 & Manufacturing, nec; recycling & $36-37$ \\
15 & Electricity, gas and water supply & $40-41$ \\
16 & Construction & 45 \\
\hline
\end{tabular}

\section{B Country coverage}

\begin{tabular}{lc}
\hline Country & I-O and ANBERD data \\
\hline Japan & 1995 \\
Korea & 1995 \\
Netherlands & 1995 \\
Poland & 1995 \\
United States & 1997 \\
\hline
\end{tabular}

